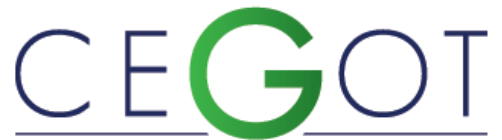

Centro de Estudos de Geografia e Ordenamento do Território
Masullo, Yata

Instituto Maranhense de Estudos Socioeconômicos e

Cartográficos - IMESC

Av. do Vale, Ed. Zircônio $1^{\circ}$ Andar Renascença II, CEP: 65075-820, São Luís MA, Brasil

yanderson3@hotmail.com

\author{
GURGEL, HELEN \\ Universidade de Brasília - UnB, Instituto de Ciências Humanas - \\ IH, Departamento de Geografia - GEA \\ CEP 70910-900, Brasília-DF, Brasil \\ helengurgel@unb.br \\ LAQUES, ANNE \\ Institut de Recherche pour le Développement (IRD), , UMR \\ ESPACE-DEV \\ 34093 Montpellier cedex 5, França \\ anne-elisabeth.laques@ird.fr
}

\title{
Métodos para avaliação da efetividade de áreas protegidas: conceitos, aplicações e limitações
}

Methods for evaluating the effectiveness of protect areas: concepts, applications and limitations

Referência: Masullo, Yata; Gurgel, Helen; Laques, Anne (2019). Métodos para avaliação da efetividade de áreas protegidas: conceitos, aplicações e limitações. Revista de Geografia e Ordenamento do Território (GOT), n. ${ }^{\circ} 16$ (março). Centro de Estudos de Geografia e Ordenamento do Território, p. 203-226, dx.doi.org/10.17127/got/2019.16.009

\section{RESUMO}

Nas últimas duas décadas surgiram metodologias e procedimentos técnicos, que visam avaliar o nível de efetividade das áreas protegidas, com o intuito de identificar a real capacidade dessas áreas em planejar e implementar políticas públicas, relacionadas a temática socioeconômica e ambiental. Nesse contexto o presente estudo, surge com a finalidade de analisar as metodologias (WWF (1999), Padovan (2003), Ervin (2003), Stolton et al. (2007), Marinelli (2011) e ICMBIO/WWF (2017), aplicadas e adaptadas à realidade brasileira, considerando estrutura metodológica, aplicação, vantagens e limitações. Dessa forma, intenciona-se avançar nas discussões metodológicas propostas e analisar o seu nível de contribuição para com o planejamento e gestão dessas áreas protegidas.

Palavras-chave: Indicadores socioambientais; Efetividade; Unidades de Conservação; Brasil.

\section{ABSTRACT}

Over the last two decades, technical methodologies and procedures have emerged to assess the effectiveness of protected areas in order to identify the real capacity of these areas to plan and implement public policies related to socioeconomic and environmental issues. In this context, the present study arose with the purpose of analyzing the methodologies (Padovan, 2003), Ervin (2003), Stolton et al. (2007), Marinelli (2011) and ICMBIO / WWF 
(2017) applied and adapted to the Brazilian reality, considering methodological structure, application, advantages and limitations. Thus, it is intended to advance in the proposed methodological discussions and to analyze their level of contribution to the planning and management of these protected areas.

Keywords: environmental indicators; Effectiveness; Protect Area; Brazil.

\section{Introdução}

O avanço das discussões sobre a avaliação da efetividade das unidades de conservação áreas protegidas, segue uma perspectiva conceitual e técnica, que as observa como um importante instrumento para implementação de políticas públicas ligadas a proteção e preservação do meio ambiente, bem como para fomentar o desenvolvimento econômico regional, mediação de conflitos, inclusão social e ordenamento territorial.

De acordo com Moraes et al. (2016), um dos grandes entraves para implementações dessas políticas públicas supracitadas, é a não efetivação dessas áreas protegidas. Os autores complementam afirmando que para ampliar as ações que possuam a capacidade de alterar esse panorama, é necessário aumentar a coleta e análise de dados que permitam a construção de metodologias, a fim de desenvolver interpretações realísticas dos fenômenos, bem como otimizar o planejamento estratégico e a gestão integrada. Essa visão, abre um leque de possibilidades para a construção e análise de indicadores e índices sintéticos ligados ao planejamento e gestão das áreas protegidas. Considerando-se que uma área protegida se torna efetiva, quando está possui capacidade e competências particulares, que permitam a essa área protegida cumprir satisfatoriamente com as funções para as quais foi criada (HOCKINGS et al., 2015).

Desse modo a partir da década de 1990, impulsionado pela Conferência das Nações Unidas e International Union For Conservation or Nature - IUCN, preocupou-se em identificar lições aprendidas no processo de avaliação da efetividade, definindo os temas que merecem maior atenção dos pesquisadores e gestores de áreas protegidas, além de identificar e desenvolver metodologias e indicadores que representem a realidade e a dinâmica local das áreas protegidas (SILVA, 2016). 
Identifica-se que a partir deste momento, há um esforço em escala global para a ampliação das discussões metodológicas e construção de indicadores ambientais para otimizar o planejamento e gestão das áreas protegidas. Isso ocorre em um cenário, onde o planejamento e gestão das áreas protegidas se complexifica, influenciado por aspectos econômicos, sociais, culturais e políticos, originários da relação conflituosa entre a sociedade e a natureza, bem como pela necessidade das pessoas em se reconectarem com ambiente. Nesse sentido o presente artigo, objetiva avaliar as metodologias desenvolvidas e aplicadas em diversos países e posteriormente adaptadas à realidade brasileira, bem como analisar as metodologias mais reproduzidas pelas instituições governamentais e de pesquisa do Brasil.

Para tanto, estruturou-se a pesquisa acerca das publicações por meio do portal de periódicos da CAPES (Coordenação de Aperfeiçoamento de Pessoal de Nível Superior), SCIELO e Google Acadêmico, a partir das palavras chaves: Indicadores socioambientais; Efetividade; Unidades de Conservação; Áreas protegidas.

Dessa forma a presente pesquisa visa contribuir com a discussão, a partir do desenvolvimento de um ensaio teórico sobre os conceitos, aplicações, vantagens e limitações das metodologias direcionadas a avaliação da gestão das unidades de conservação - UCs, (Sistema de Certificação de Áreas Protegidas, Rapid Assessment Priorization Protected Area - RAPPAM, Management Effectiveness Tracking Tool - METT, Grau de Implementação e Vulnerabilidade de Unidades de Conservação, Sistema de Indicadores Socioambientais de Unidades de Conservação - SISUC e Sistema de Análise e Monitoramento de Gestão - SAMGe).

\section{Contribuições e perspectivas para a construção de metodologias de avaliação da efetividade das unidades de conservação: conceito, aplicações e limitações}

Na última década, foram realizadas análises de lacunas globais e regionais que tiveram como objetivo principal, informar a situação dos sistemas de áreas protegidas para a 
UICN/WCPA e de como o planejamento territorial influencia na efetividade dessas unidades (JUFFE-BIGNOLI et al., 2014). Segundo os autores entre os principais gargalos identificados está o descaso do poder público com o planejamento desses territórios ou mesmo da ausência de conexão dessas metodologias com a realidade.

De acordo com Wilkie et al. (2006) corrobora para essa realidade, a grande diversidade de indicadores ${ }^{1}$ e índices ${ }^{2}$, o que nos leva a refletir sobre a impossibilidade de se vislumbrar formas de mensurar a efetividade das UCs, que possam vir a ter ampla aceitação, mesmo com as limitações relacionadas à incipiência dos dados, além das discordâncias metodológicas e conceituais.

Nesse contexto Campedelli et al. (2010), afirmam que o desenvolvimento de metodologias associadas às tecnologias e as diferentes realidades territoriais das áreas protegidas, tornam possível à busca constante por melhores soluções aos desafios interligados a criação e efetivação de políticas públicas. Possibilita-se dessa forma a redução da subjetividade, além de determinar a capacidade de uma metodologia em fornecer as respostas certas a uma dada questão. Entretanto, a simples avaliação de eficácia da gestão através da aplicação de indicadores e índices, embora necessária, poderá não ser suficiente para mensurar o quanto dos objetivos da área protegida foram atingidos (HOCKINGS et al., 2015).

Via de regra, um dos pressupostos da ciência é a refutação de hipóteses e teorias (MORIN et al., 2003). Dessa forma, métodos de como analisar os padrões da natureza ou como manejar a biodiversidade são constantemente reavaliados ou complementados. Percebe-se que os modelos produzidos até o momento, devem ser reelaborados e revistos na tentativa de ampliar a aproximação com a realidade (PADUA e CHIARAVALLOTI, 2012).

Seguindo esses princípios, diversas experiências no mundo e no Brasil foram direcionadas para a construção e consolidação de metodologias, que possibilitassem a mensuração e representação da efetividade das áreas protegidas em escala local e regional (Quadro 01). A

\footnotetext{
${ }^{1}$ Os indicadores podem ser reconhecidos como modelos simplificados da realidade, com a capacidade de possibilitar a compreensão dos fenômenos e ampliar a interpretação e comunicação de dados brutos (SOBRAL et al, 2011).

${ }^{2}$ Os índices sintéticos são constituídos por um conjunto de variáveis e indicadores que, submetidos a operações estatísticas transforma o dado em informação, com a capacidade de aglutinar questões complexas. Isso possibilita a simplificação da informação, identificação de tendências e padrões de indicadores individualizados, além de repassar a informação de forma didática (JANUZZI, 2006).
} 
partir de uma visão geral dos instrumentos de avaliação da eficácia em áreas protegidas, foram desenvolvidos cerca de cinquenta metodologias diferentes na África, Ásia, América e Europa (GETZNER et al, 2012).

\begin{tabular}{|c|c|c|}
\hline METODOLOGIA & ORGANIZAÇÃO & PERÍODO DE APLICAÇÃO \\
\hline $\begin{array}{c}\text { Implementação e } \\
\text { Vulnerabilidade de Unidades de } \\
\text { Conservação }\end{array}$ & WWF Brasil & 1999 \\
\hline $\begin{array}{l}\text { Certificação de Unidades de } \\
\text { Conservação - Parâmetros e } \\
\text { procedimentos para certificação } \\
\text { do manejo de unidades de } \\
\text { conservação }\end{array}$ & $\begin{array}{l}\text { Instituto de Pesquisas } \\
\text { da Mata Atlântica - } \\
\text { IPEMA }\end{array}$ & 2002 \\
\hline Scenery Matrix & $\begin{array}{l}\text { Instituto Florestal do } \\
\text { Estado de São Paulo }\end{array}$ & $1999-2005$ \\
\hline Parkswatch & $\begin{array}{l}\text { Centro de Conservação } \\
\text { Tropical da } \\
\text { Universidade de Duke e } \\
\text { Fundação O Boticário }\end{array}$ & $2002-2006$ \\
\hline $\begin{array}{l}\text { Indicadores da Efetividade de } \\
\text { Implementação (IEI) das UCs } \\
\text { estaduais }\end{array}$ & SDS, Amazonas & 2006 \\
\hline $\begin{array}{l}\text { Programa de Monitoramento da } \\
\text { Biodiversidade e do Uso de } \\
\text { Recursos Naturais em Unidades } \\
\text { de Conservação Estaduais do } \\
\text { Amazonas (PROBUC) }\end{array}$ & SDS, Amazonas & 2006 \\
\hline $\begin{array}{c}\text { Protocolo de avaliação de } \\
\text { efetividade de gestão de } \\
\text { mosaicos de áreas protegidas no } \\
\text { Brasil }\end{array}$ & Gidsicki, D. & 2013 \\
\hline RAPPAM & WWF Brasil e ICMBIO & $2005-2010-2015$ \\
\hline $\begin{array}{l}\text { FAUC - Ferramenta de Avaliação } \\
\text { da Efetividade do Programa } \\
\text { Áreas Protegidas da Amazônia }\end{array}$ & $\begin{array}{c}\text { Programa áreas } \\
\text { Protegidas da Amazônia } \\
\text { - Arpa }\end{array}$ & $2006-2015$ \\
\hline METT & Banco Munidal e WWF & $\begin{array}{l}2005-2006 \\
2010-2015\end{array}$ \\
\hline SISUC & Instituto Socioambiental & 2011 \\
\hline Avaliação de Mosaico & WWF & 2015 \\
\hline SAMGe & ICMBIO/WWF Brasil & $2015-2016$ \\
\hline
\end{tabular}

Quadro 01 - Metodologias utilizadas no Brasil para avaliação da efetividade de unidades de conservação.

Fonte - Adaptado Silva, 2016; ICMBIO/WWF, 2017; UICN, 2018.

Mesmo com essas diversas metodologias desenvolvidas, Hockings et al. (2015) afirma que ainda são poucos os países ou agências que estão avaliando com periodicidade regular o nível de efetividade das áreas protegidas. Autores como Leverington et al. (2010); StollKleemann (2010); Nelson e Chomitz (2011), Geldmann et al. (2014) e Silva (2016) ressaltam que essas ferramentas diferem entre si, em relação a abordagem conceitual, aos objetivos 
de aplicação, a forma de aplicação, a área de abrangência, aos indicadores, aos critérios de análise, a forma e aos softwares utilizados para o cálculo de efetividade.

Observa-se que estes e outros estudos relacionados com a avaliação da efetividade de UCs, reforçaram que seria altamente vantajoso sintetizar informações específicas do local, para obter maior compreensão dos fatores de sucesso nas abordagens de conservação e integrar estudos em nível local. Entretanto, segundo o WWF (2017), definir até que ponto as UCs ou o sistema destas são efetivos, tem sido uma árdua tarefa expressada pela aplicação de diversas ferramentas.

Para Geldmam et al. (2017) os métodos de avaliação da efetividade das áreas protegidas em geral, demonstram que há boas evidências de que esses territórios conservam o habitat florestal, mas que ainda são inconclusivas a contribuição destas, sobre os impactos socioeconômicos associados a gestão das mesmas. Para tanto, é preciso alterar o foco, pois a avaliação do impacto aborda o fim, não os meios da conservação da natureza.

Diante desses novos métodos e pressupostos científicos, observa-se a necessidade do desenvolvimento de análises mais precisas sobre a realidade. Tendo em vista que métodos mais modernos, permitem um menor esforço físico/monetário e com resultados mais precisos, fato de grande importância diante dos poucos recursos destinados aos gestores e da necessidade de respostas rápidas para a sociedade (PADUA e CHIARAVALLOTI, 2012).

Segundo Silva (2016), essas metodologias devem utilizar modelos empíricos robustos, com um conjunto amplo de dados para estimar os efeitos que as áreas protegidas têm sobre os resultados ambientais e sociais. Essa simples, mas complexa diferença possilita alcançar segundo a autora a construção de um sistema efetivamente representativo e manejado, além de apresentar uma forma de se verificar que o investimento de tempo e esforço, na criação e gestão dessas áreas resultam nos benefícios que a sociedade procura.

A autora ainda ressalta, que para o desenvolvimento de metodologias para a avaliação da efetividade de UCS, o marco conceitual deve ser utilizado como ponto de partida para adaptação das metodologias existentes, combinando diferentes procedimentos e abordagens. A seguir analisa-se as metodologias de avaliação de efetividade mais reproduzidas no Brasil, considerando seus avanços, limitações, indicadores e procedimentos metodológicos. 


\subsection{Rapid Assessment Priorization Protected Area - RAPPAM}

Aplicado em aproximadamente 150 países o método RAPPAM foi desenvolvido pelo WWF, como uma metodologia de rápida avaliação da efetividade de gestão das áreas protegidas (ERVIN, 2003). Com o objetivo geral de analisar o nível de efetividade da gestão das áreas protegidas, este método foi empregado em diversos países como Indonésia, Gana, Chile, Butão, Brasil, China, Romênia, Rússia, África do Sul, Bulgária, Senegal e Nova Guiné conforme o ICMBio (2011).

No Brasil de acordo com Banzato (2014), o ICMBio avaliou o nível da efetividade de 260 UCs federais em 2005/2006 e em 2010, a avaliação foi replicada com base nessa metodologia, abrangendo 292 UC. Já em 2015, o estudo alcançou 260 federais e estaduais (110 unidades de conservação federais, 39 de proteção integral e 71 de uso sustentável, já no âmbito estadual, o RAPPAM foi aplicado em 40 unidades (19 de proteção integral, 20 de uso sustentável e no mosaico, Mosaico Apuí no Amazonas) (WWF, 2017).

Seguindo uma abordagem qualitativa da gestão empregada na UC, o ciclo de atualização da metodologia ocorre a cada 5 anos, por meio de formulário eletrônico, baseada nas informações cedidas pelo gestor da UC e validado pelo órgão gestor. Os questionários consideram cinco elementos (Figura 01).

Contexto: evidenciar o cenário atual em que se encontra a UC, considerando o objetivo, tamanho, equipe de trabalho e tempo de criação;

Planejamento: avalia informações sobre seu objetivo, amparo legal, desenho e planejamento territorial;

Insumos: analisa os recursos humanos, de comunicação e informação, infraestrutura e financeiros;

Processos: avalia o planejamento da gestão, a tomada de decisão e o desenvolvimento de pesquisa, avaliação e monitoramento realizados na UC;

Resultados: evidencia as ações desenvolvidas nos dois anos anteriores à data da aplicação do questionário.

Figura 01 - Elementos para avaliação da efetividade de UC, utilizados pelo RAPPAM.

Fonte - Adaptado Ervin, 2003.

De acordo com Silva (2016), o método RAPPAM é adequado para análise integrada do conjunto de áreas, entretanto o método não foi elaborado para gerar orientações 
específicas para cada gestor de unidade. O índice criado é expresso como um índice percentual, equivalente a um valor proporcional da efetividade de gestão observada em relação à efetividade máxima.

O RAPPAM estruturou-se a partir da aplicação de questionários em oficinas participativas, com foco nos gestores das UCs; equipe técnica da sede do ICMBio; consultores especializados na metodologia e equipe técnica do WWF-Brasil. Este índice é calculado com base nos resultados destes elementos, ponderados pela pontuação máxima possível para esse conjunto de respostas realizada por pessoal selecionado, como pontos focais nas regionais do ICMBio e nos estados participantes do Programa de Áreas Protegidas da Amazônia - ARPA (WWF, 2017). Para construção do índice foram sistematizadas através da capacitação dos pontos focais; preenchimento dos questionários, com apoio dos pontos focais para sistematização dos questionários e apresentação dos resultados.

A avaliação da gestão desenvolvida pelo RAPPAM, trabalha com base em uma abordagem sistêmica do processo, por entender que não se pode abstrair a influência da realidade que envolve as unidades de conservação. Dessa forma, o processo se inicia pela análise do contexto em que as áreas protegidas se inserem e, por isso, considera informações sobre a importância biológica e socioeconômica, as pressões e ameaças que as afetam e o nível de vulnerabilidade existente (WWF e SEMARH-GO, 2014) (Figura 02).

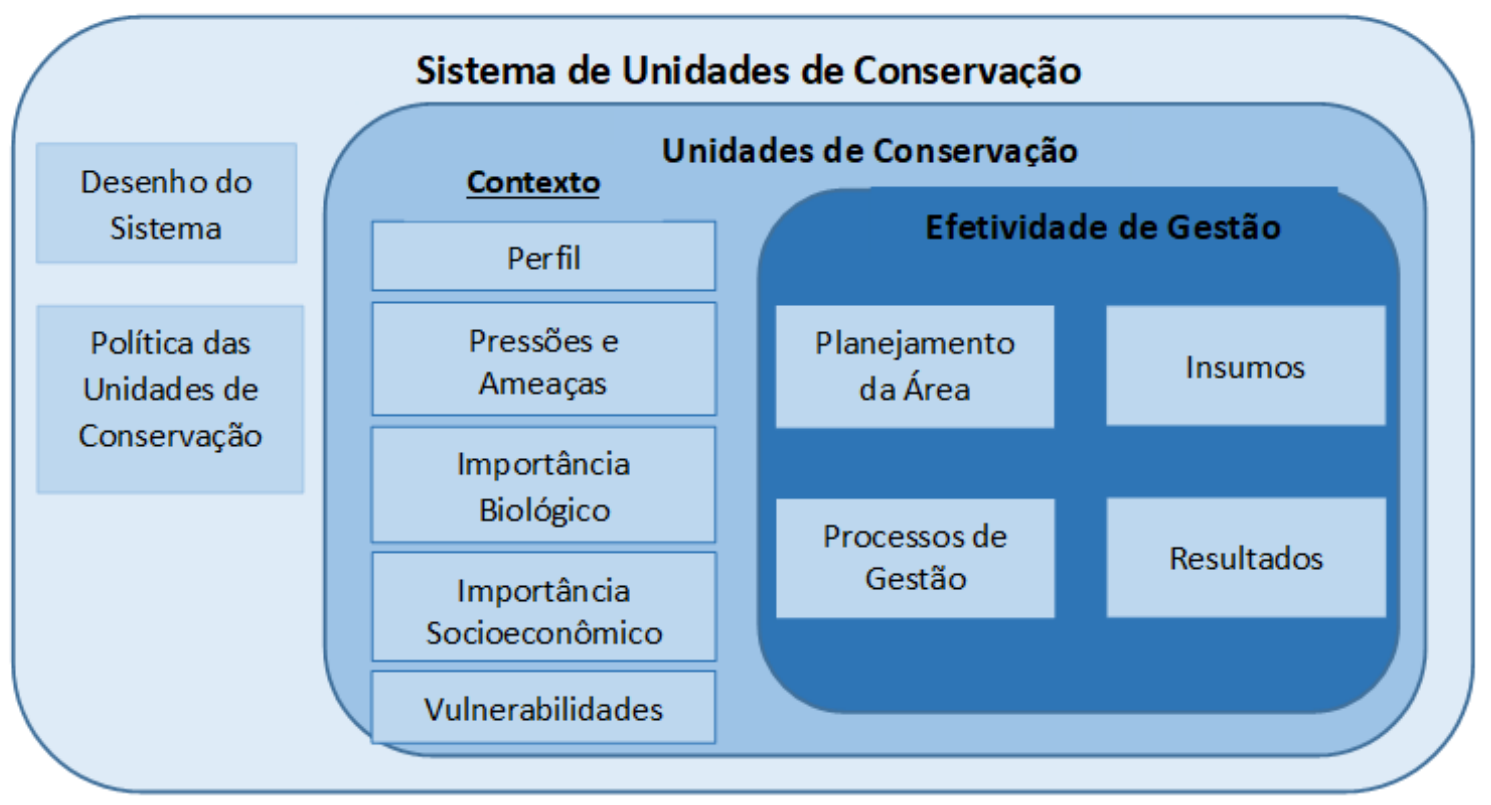

Figura 02 - Estrutura do Questionário do Método RAPPAM.

Fonte - ICMBio/WWF Brasil, 2017. 
Silva (2016) ressalta que a pressão foi avaliada, com base na ocorrência de impactos negativos que influenciaram a integridade da UC, considerando a probabilidade de ameaças ocorrerem no curto prazo. Outro fator considerado foi o grau de permanência (tempo em anos de persistência das pressões e das ameaças) e o nível de severidade que essas pressões e ameaças são percebidas pelo gestor da UC. O nível de criticidade, foi mensurado a partir da multiplicação da pontuação dos atributos abrangência, impacto e permanência das pressões ou ameaças. Basicamente, as análises das pressões e ameaças se baseiam na identificação de sua ocorrência, tendência, extensão, grau de impacto e permanência. Posteriormente, estes dados são hierarquizados em uma escala de pontuação graduada, onde se estabeleceu o nível de efetividade (baixa, média e alta) (ICMBIO, 2011).

De forma geral, segue abaixo a escala de valorização utilizada para mensurar o nível de efetividade das UCs (Quadro 02).

\begin{tabular}{|c|c|}
\hline Variação & Nível de efetividade \\
\hline$<40 \%$ & Baixa \\
\hline $\mathbf{4 0 \%}$ a $\leq 60 \%$ & Média \\
\hline$>60 \%$ & Alta \\
\hline
\end{tabular}

Quadro 02 - Escala de Valorização utilizada para mensurar o nível de efetividade das UCs. Fonte - WWF, 2017.

A valoração da efetividade de gestão, é obtida pela soma das respostas de cada um dos módulos que compõem esses elementos. Segundo a metodologia aplicada, observou-se que entre as UCs de proteção integral analisadas entre 2005 a 2015, houve um aumento na efetividade de gestão na faixa média de classificação de $41 \%$ para $54 \%$. Em relação as UCs de uso sustentável, o nível de efetividade aumentou de 2005 (35\%) para 2015 (56\%).

Segundo o WWF e Semarh-GO (2014), esse método pode auxiliar na identificação das áreas que precisam de estudos mais detalhados, identificando os pontos fortes e fracos do manejo; avaliar as características e a distribuição das diversas ameaças e pressões; identificar áreas de alta importância socioecológica e vulnerabilidades; indicar prioridades na gestão das UCs e nas intervenções políticas para o desenvolvimento de ferramentas de monitoramento local, além de fornecer critérios para a elaboração de indicadores específicos. 
Entretanto para Marinelli (2011), essa metodologia apresenta limitações, por restringir a correlação entre os aspectos socioeconômicos e socioculturais. Para Júnior e Agra Filho (2015) a aplicação do RAPPAM visa o estabelecimento de prioridades para o planejamento, sendo assim, os autores desaconselham que essa metodologia seja aplicada sobre áreas protegidas de forma isolada, dado o seu alto poder de comparação decorrente da generalização dos indicadores utilizados para aferir o grau de efetividade de cada elemento indicado pelo UICN-WCPA. Outro fato limitante segundo Banzato (2014), se refere as aplicações do questionário, focados no chefe da UC, o que não possibilita a visualização completa da gestão.

\subsection{Management Effectiveness Tracking Tool - METT}

Aplicado em cerca de oitenta e cinco países, o METT foi desenvolvido pela parceria entre Banco Mundial e WWF. Atualmente é utilizado principalmente por agências e ONGs, como um instrumento para identificar necessidades, restrições e ações prioritárias para melhorar a eficácia da gestão de áreas protegidas na Europa, Ásia, África e América Latina, principalmente em áreas de atuação do Banco Mundial (STOLTON et al., 2007; JÚNIOR e AGRA FILHO, 2015).

Juntamente com o RAPPAM, a metodolgia do METT está entre as mais utilizadas em nível mundial. Ambas as metodologias foram baseadas em conhecimentos especializados e avaliações qualitativas, especialmente com ênfase na avaliação rápida (SILVA, 2016). Em outro contexto, o METT foi estruturado para permitir maior eficiência na prestação de contas quanto aos investimentos realizados, enquanto que o RAPPAM, possui o foco no fornecimento de informações para a priorização e alocação de recursos.

O METT foi projetado para ser simples e implementado com baixos custos, através da aplicação de questionários com gestores da área protegida e um representante da comunidade local. No formulário são feitas trinta perguntas, que posteriormente são hieraquizadas a partir da escala de pontos abaixo (Figura 03). 


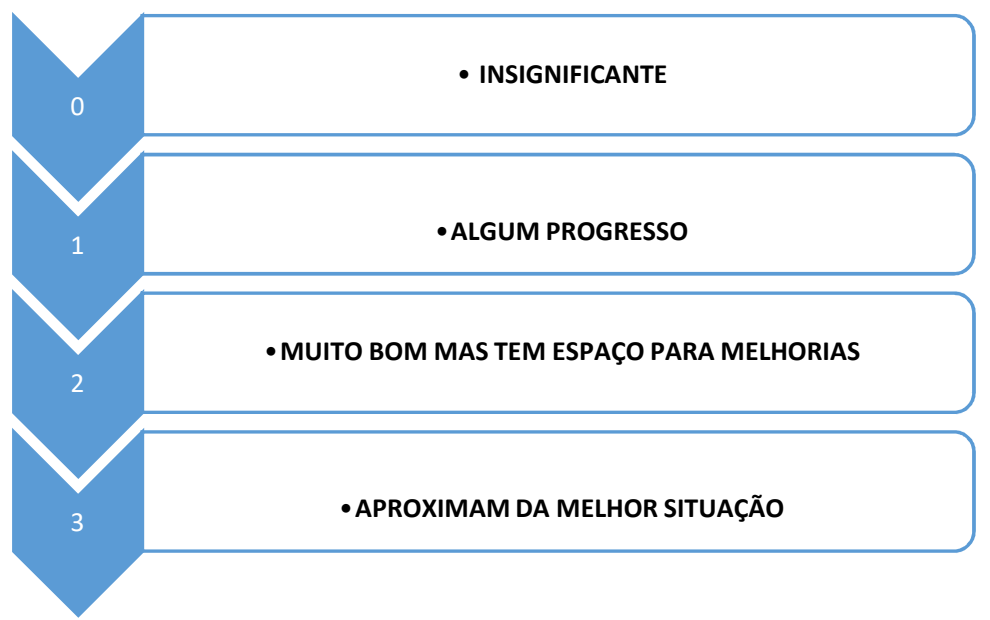

Figura 03 - Valoração utilizada pelo METT para avaliação da efetividade de unidades de conservação Fonte - adaptado Stolton et al., 2007.

De acordo com Júnior e Agra Filho (2015) o método METT, fornece a possibilidade de acompanhamento do progresso da efetividade de gestão ao longo do tempo. Mais do que para comparar áreas protegidas, a metodologia revela tendências, aspectos favoráveis e fraquezas. Além disso, os autores ressaltam que a estrutura do questionário, torna seu preenchimento simples e rápido, o que possibilitou uma grande quantidade de avaliações e consolidação do banco de dados, sobre as tendências internacionais na gestão de áreas protegidas.

De acordo com Stoll-Kleemann (2010), quanto à efetividade o METT produz resultados contraditórios ao RAPPAM. Isso se deve, a aspectos ligados a abordagem desses métodos, principalmente, considerando as informações baseadas nos gestores das unidades no que diz respeito as singularidades territoriais e o contexto local e regional.

Nota-se que apesar da simplificação da coleta das informações, identifica-se distorções em relação ao cálculo da significância do dado. De acordo com Stolton et al. (2007) a hierarquização e pontuação das informações fornecem uma melhor avaliação da eficácia, se calculada como uma porcentagem para cada um dos seis elementos indicados pelo International Union for the Conservation of Nature (IUCN)/World Commission on Protected Areas (WCPA) (contexto, planejamento, insumos, processo, resultados e avaliações), correlacionando diferentes dimensões de análise. 


\subsection{Sistema de Indicadores Socioambientais de Unidades de Conservação (SISUC)}

Desenvolvida pelo Instituto Socioambiental - ISA, o SISUC é uma metodologia de avaliação e monitoramento estratégico de UCs. Este método segue uma abordagem socioambiental, adotando conceitos práticos, além de seguir princípios como multiplicidade, integrabilidade, complementaridade, comunicabilidade, transparência e continuidade. Esse sistema, adota indicadores de processos socioambientais e busca a integração com a comunidade local, através de fóruns participativos (MARINELLI, 2011).

Criado em 2008, como uma ferramenta para auxiliar a gestão das UCs, sua aplicação se deu inicialmente na região norte do País, junto aos conselhos destas unidades (SILVA, 2016). A metodologia foi aplicada inicialmente nas UCs de uso sustentável e proteção integral das áreas protegidas do baixo rio Negro, no estado do Amazonas. A metodologia estrutura-se em seis etapas: qualificação de indicadores construídos no workshop; construção de protocolo de aplicação da metodologia; aplicação-piloto do protocolo; avaliação da metodologia; análise de resultados e definição de protocolo.

Conforme Costa e Marchand (2014), o SISUC representa a criação de um sistema de avaliação e monitoramento da gestão das UCS, localizadas na região amazônica brasileira, a partir da construção de uma metodologia coletiva e participativa. A metodologia em análise, utiliza-se de indicadores nas dimensões econômica, sociocultural, ambiental e gestão (Figura 04). 


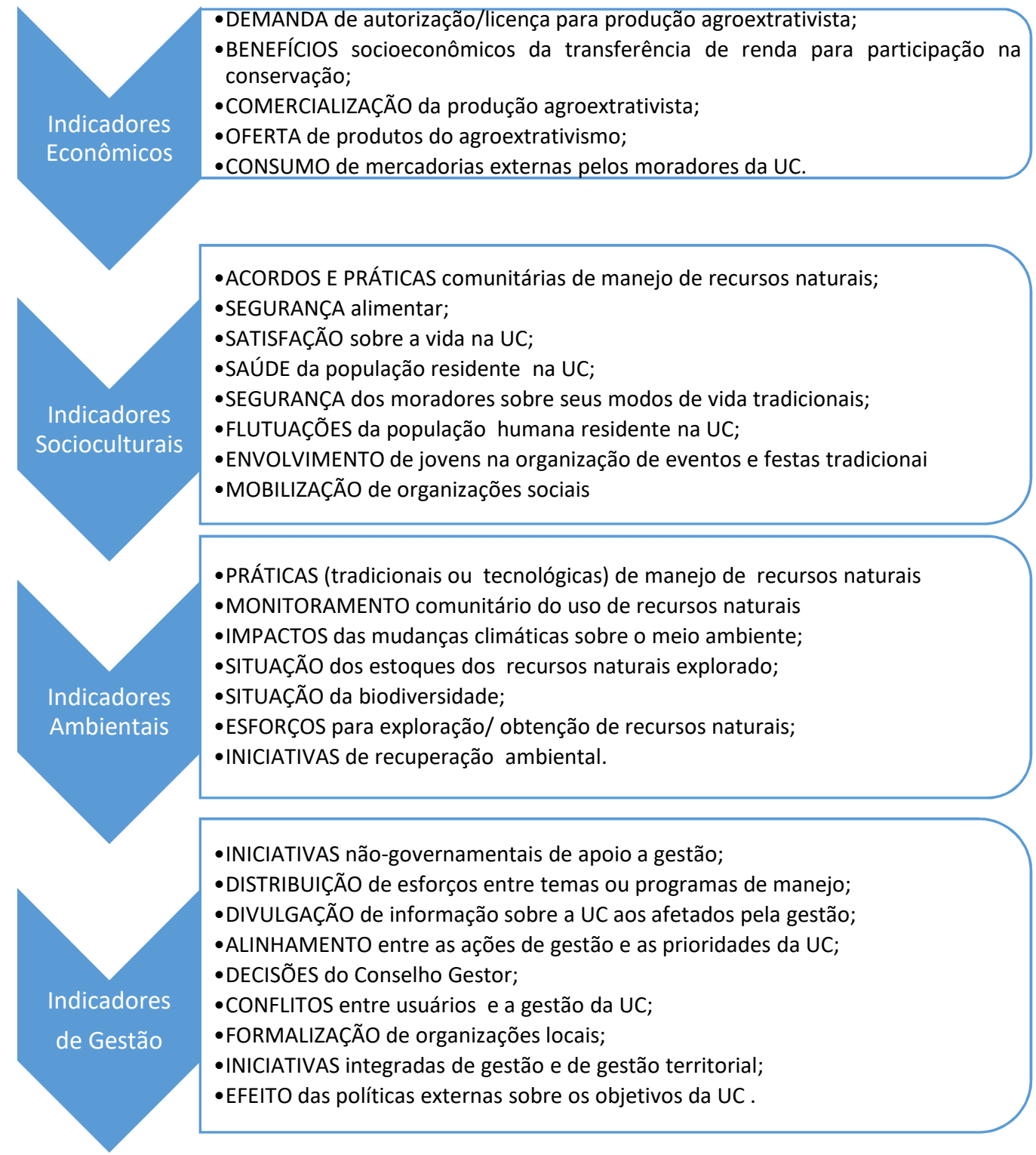

Figura 04 - Indicadores utilizados pelo SISUC para avaliação da efetividade de unidades de conservação

Fonte - Adaptado Marinelli, 2011.

A dimensão econômica utiliza-se indicadores referentes à produção agroextrativista e consumo local; sociocultural foca no bem-estar humano como aspectos relacionados a saúde, segurança, nível de satisfação sobre a vida e o envolvimento na organização local; ambiental com alvo nos serviços e na conservação ambiental, considerando práticas de manejo, alterações ambientais, situação da biodiversidade e iniciativas de recuperação 
ambiental. Além de indicadores ligados a gestão, que relacionam as ações de gestão pública e da sociedade, assim como a efetivação de políticas públicas.

A metodologia foi aplicada através de fóruns participativos, que obtiveram participação de cerca de 500 pessoas e 80 instituições. Segundo Silva (2016), os resultados obtidos nas oficinas fomentam a ampliação do conhecimento da sociedade sobre a situação socioambiental da UC, bem como fornece subsídios a elaboração de acordos e de planos de manejo pautados no desenvolvimento socioambiental local; alertas preditivos para priorização e ajuste de ações, projetos e programas; comparar resultados entre UCs inseridas no mesmo contexto regional e para fomentar ações estratégicas no âmbito de conjuntos de UCs.

Com a finalidade de representar os vetores resultantes de interações entre variáveis socioambientais, a metodologia engloba 29 indicadores distribuídos em 4 categorias, economia (5), meio ambiente (7), sociocultural (8) e gestão de UC (9). As informações são obtidas através de questionários aplicados com os membros do Conselho da UC, diferenciando do RAPPAM nesse aspecto, mas igualmente baseia-se na percepção e subjetividade dos respondentes, ponderados nesta ferramenta pela percepção do Conselho Gestor (SILVA, 2016).

Entretanto, ressalta-se que os indicadores construídos apresentam baixa capacidade de reprodução, resultando em um elevado custo para a coleta de dados, o que dificulta a avaliação de forma sistêmica e com periodicidade relevante. Outra questão identificada em estudo desenvolvido pela autora supracitada, refere-se a metodologia, que mesmo buscando grande participação popular, reproduções da metodologia registraram reduzida participação dos conselheiros nas oficinas. A partir dessa realidade considera-se que participar de reuniões públicas representa custos não só para o setor público, mas para os indivíduos, bem como investimento de tempo. Não ponderar sobre esses elementos, propicia a redução considerável da participação popular, que demanda definições e o retorno por parte dos órgãos públicos. 


\subsection{Grau de implementação e vulnerabilidade de unidades de conservação}

A metodologia de avaliação do nível de efetividade das UCs foi implementada no Brasil em 1998, organizada pela WWF- Brasil, com a finalidade de analisar a situação de todas as unidades de conservação federais de uso indireto com mais de 6 anos de criação, sendo 86 (oitenta e seis) unidades analisadas, entre elas: Estações Ecológicas, Parques Nacionais, Reservas Biológicas, Reserva Ecológica (WWF, 2000). As instituições responsáveis pelo estudo, formaram uma equipe conjunta para planejar e gerenciar a avaliação.

Foram selecionados oito indicadores-chave para mensurar a implementação das áreas protegidas: situação fundiária; demarcação de fronteira; existência de plano de manejo; presença ou ausência de atividades ilegais dentro da área protegida; orçamento recebido em relação ao orçamento solicitado; número de pessoal; adequação dos equipamentos e infra-estruturas.

Dois workshops conjuntos foram realizados com a participação de técnicos do WWF e do IBAMA, além de especialistas convidados. O estudo avaliou a situação das UCs federais de uso indireto, determinando a extensão dos problemas e a priorização das ações. Para tanto, foram selecionados cinco indicadores para medir a vulnerabilidade da área protegida: grau de fragmentação; percentual de área degradada dentro da área protegida; exploração ilegal de recursos intra UC e presença de grandes projetos na zona de entorno (LEMOS DE SÁ, 2000).

Ressalta-se que todos os dados coletados, foram cedidos por gestores das unidades através do preenchimento de questionários. Como a maior parte das questões são quantitativas, reduz-se a subjetividade das respostas, além de permitir uma melhor padronização das mesmas.

As análises da implementação e da vulnerabilidade, foram utilizadas para preparar uma "matriz de risco" que agrupa a conservação de acordo com as classificações e o grau de risco de cada área (HOCKINGS et al., 2000). De acordo com o WWF (2000), a pontuação de cada resposta do formulário de coleta varia de 0 a 4, usando-se o seguinte critério (resposta 1 equivalente a 4 pontos, resposta 2 equivalente a 3 pontos, resposta 3 equivalente a 2 pontos, resposta 4 equivalente a 1 ponto e resposta 5 equivalente a 0 ponto). 0 grau de 
implementação e de vulnerabilidade das unidades de conservação, foi obtido pela média aritmética das questões respondidas no formulário.

De acordo com o estudo, identificou-se que das 86 unidades federais analisadas, apenas 8,4\% cumprem razoavelmente o seu papel de conservação da natureza. Isso significa que a rigor, apenas 7 das unidades de conservação analisadas são razoavelmente implementadas, ou seja, estão aptas a enfrentar satisfatoriamente as crescentes pressões externas e internas enfrentadas por áreas naturais. Enquanto que 32 (37\%), atendem apenas aos requisitos mínimos necessários. As demais 47 áreas (55\%) estão em situação precária ou praticamente inexistente.

O relatório ressalta ainda, que não há qualquer unidade de conservação federal de uso indireto no Brasil plenamente implementada. O estudo complementa afirmando que $62 \%$ das UCs no Brasil, apresentam uso incompatível com a finalidade prevista em lei. Enquanto que $45 \%$ destas, possuem menos da metade dos recursos financeiros necessários para a sua implementação, $28 \%$ não possuem infraestrutura adequada e igual percentual têm menos da metade de sua área regularizada.

Os autores desenvolveram uma análise conjunta dos dados relativos à implementação e vulnerabilidade, o que permitiu a elaboração de uma "matriz de risco", que agrupou as 86 unidades em quatro blocos, segundo o grau de ameaça enfrentado (quanto maior for a vulnerabilidade e menor for a implementação, maior o risco que a UC está correndo). Essa matriz de risco elaborada, demonstrou a situação das UCs brasileiras (Quadro 03).

\begin{tabular}{|c|c|}
\hline Grau de Risco & $\begin{array}{c}\text { Percentual das Unidades de } \\
\text { Conservação }\end{array}$ \\
\hline Risco extremo & $23 \%$ \\
\hline Risco alto & $20 \%$ \\
\hline Risco mediano & $31 \%$ \\
\hline Risco normal & $26 \%$ \\
\hline
\end{tabular}

Quadro 03 - Grau de risco das unidades de conservação federal Fonte - WWF, 2000. 
Lemos de Sá et al. (2000), afirma que este estudo marca o início de uma análise padronizada da situação real das UCs federais do Brasil, gerando dados que poderão servir de guia para ações concretas de conservação da biodiversidade, bem como permitir a otimização do monitoramento dessas áreas, a partir de futuros estudos. Os autores ressaltam que essa metodologia também pode ser aplicada em UCs estaduais e municipais, ampliando a abrangência do trabalho.

Contudo para Mori e Christodoulou (2012), o estudo desenvolvido pela WWF apresenta limitações no formulário em relação à coleta de dados referentes a biodiversidade, tendo em vista que este parâmetro não possui uniformidade entre as UCs. Se essa informação fosse coletada de forma sistemática através da metodologia empregada, haveria elevação dos custos da coleta de dados e inviabilização do estudo.

Outro fator limitante identificado, foi a divergência identificada entre as informações obtidas no ICMBio de Brasília e do chefe da unidade local, além das diferenças de interpretação de cada gestor ao responder as perguntas. Lemos de Sá et al. (2000) complementa, afirmando que alguns membros do IBAMA responderam de forma defensiva, observando que havia um foco na sua gestão e não nas difíceis circunstâncias enfrentadas pelos gerentes de áreas protegidas no Brasil. Portanto, é possível que algumas respostas tenham sido superestimadas ou mesmo subestimadas (WWF, 2000).

\subsection{Sistema de certificação de áreas protegidas}

O estudo de certificação do manejo de áreas protegidas, foi estruturado por meio da coleta de dados sistemática sob aspectos ambientais, sociais, econômicos e institucionais. Com o objetivo de desenvolver e consolidar uma ferramenta capaz de avaliar o nível de efetividade das UCs. Para Padovan (2003), o estudo possibilita aos administradores das UCs públicas ou privadas, as bases para orientar a tomada de decisões e guiar o manejo da área de forma compatível com os usos permitidos para a categoria de manejo, através de monitoramento sistemático, contribuindo para a profissionalização da gestão e para o manejo adaptativo.

O primeiro teste da metodologia de estudo ocorreu em três áreas protegidas: Reserva Biológica de Monteverde na Costa Rica; Reserva da Biosfera do Rio Plátano, em Honduras; e Parque Nacional de Tikal, na Guatemala, posteriormente este método de avaliação foi 
aplicado em UCs inseridas na Mata Atlântica e no nordeste brasileiro. Para Cifuentes e Izurieta (1999), a metodologia em análise possibilita aplicação regular por parte do poder público, por ser de simples aplicação, além de ser baseada em um sistema de pontuação desenvolvido para atender as necessidades especiais das áreas protegidas na América Latina.

Essa metodologia foi consolidada a partir de 7 princípios, 26 critérios e 67 indicadores organizados nas áreas anteriormente referidas (PADOVAN, 2003). Para agregação dos dados, utiliza-se uma escala de valorização baseada no ISO 10.004, cuja poderação percentual é hierarquizada de 0 a 4 (Figura 05).

- Insatisfatório: corresponde a uma área protegida com grande carência de recursos básicos sem o manejo necessário, não garantindo a sua conservação em médio prazo.

- Pouco Satisfatório: mostra uma área protegida sendo gerida com poucos recursos para estabelecer o manejo e com alta condição de vulnerabilidade socioambiental.

- Medianamente Satisfatório: apresenta os requerimentos mínimos para o manejo da unidade de conservação com deficiências, mas que permitem estabelecer uma base sólida de gestão mesmo que parcial.

- Satisfatório: indica que as atividade de manejo na unidades de conservação estão 3 sendo atendidas adequadamente, garantindo conservação da área a médio prazo.

- Muito Satisfatório: apresenta uma área protegida que possui um manejo eficiente, garantindo o alcance dos objetivos da unidade e sua conservação em um longo prazo.

Figura 05 - Escala de significância utilizada pelo método de Certificação de Áreas Protegidas Fonte: - Adaptado Padovan, 2001.

O estabelecimento da pontuação na metodologia, permite a comparação entre cenários e o desenvolvimento do processo de evolução. Isso possibilita o reconhecimento dos pontos fortes e fracos dos modelos de gestão instituídos. Ressalta-se que este processo de certificação utiliza parâmetros de referência previamente estabelecidos, definindo os níveis de desempenho que a UCs devem cumprir, de acordo com o seu instrumento de criação, a categoria de manejo e os objetivos estabelecidos pela UICN/WCPA. 


\subsection{Sistema de Análise e Monitoramento de Gestão - SAMGe}

O SAMGe é uma metodologia de avaliação e monitoramento de gestão, de aplicação rápida, concebida pelo ICMBio, com o apoio operacional do WW-Brasil e do Programa Arpa, e financeiro da Fundação MOORE. Seu escopo e aplicabilidade, está ligado ao marco referencial de avaliação da efetividade da WCPA/UICN. De acordo com Silva (2016), essa metodologia visa subsidiar a tomada de decisão e aproximar a sociedade da gestão das áreas especialmente protegidas por meio das mais diversas formas, como o preenchimento em conselhos, a visualização de informações e a divulgação de resultados. O SAMGe busca mensurar a efetividade de gestão das UCs, a partir da análise das inter-relações entre os objetivos de conservação, os usos, além de analisar o estado de conservação e alteração da paisagem, e as ações de manejo realizadas pelo órgão gestor (WWF, 2017).

Aplicada pelo ICMBio, essa metodologia analisa o nível da efetividade das UCs com base nos elementos de Contexto, Produtos e Serviços e Resultados. Já a análise de gestão interrelaciona o impacto com os elementos Planejamento, Insumos e Processos (WWF, 2017). De acordo com o ICMBio (2015) a ferramenta sugere a criação de cenários (econômicos, sociais, conservação e manejo) dos impactos positivos e negativos, com atribuição de valores.

Para qualificação das informações foi necessário identificar a abrangência do impacto: individuais; entorno ou determinado grupo de pessoas; para a sociedade como um todo. $\mathrm{Na}$ avaliação dos impactos negativos foram sugeridos cenários de severidade e magnitude, onde o gestor seleciona o valor de 0 a 5 , identificando o que melhor represente o uso na UC. O SAMGe utiliza-se de 3 níveis de enquadramento de valoração (Quadro 04). 


\begin{tabular}{|c|c|}
\hline Nível de Efetividade & Características \\
\hline Alta & $\begin{array}{l}\text { Com as políticas públicas funcionando e com a } \\
\text { execução de ações de gestão e manejo superando as } \\
\text { expectativas da sociedade. }\end{array}$ \\
\hline Moderada & $\begin{array}{l}\text { Quando os objetivos de criação da UC se } \\
\text { encontrarem em patamares mínimos para a sua } \\
\text { conservação, com situação claramente alinhada com } \\
\text { a atual conjuntura da gestão. }\end{array}$ \\
\hline Baixa & $\begin{array}{l}\text { A UC encontrar-se em situação de dificuldade na } \\
\text { gestão dos seus objetivo e apresenta um baixo } \\
\text { desempenho de retorno da política pública para a } \\
\text { sociedada }\end{array}$ \\
\hline
\end{tabular}

Quadro 04 - Escala de valorização de efetividade do SAMGe.

Fonte - WWF, 2017.

A aplicação do SAMGe, conseguiu obter respostas de 168 unidades. Já no processo de consolidação das informações, 12 UCs não foram consideradas por não conterem informações essenciais para análise mínima, sendo então contabilizadas 156 unidades, correspondendo a 48,75\% das UC Federais (WWF, 2017). O estudo identificou que de modo geral, os usos que mais influenciam negativamente o nível de efetividade são os que advêm do uso de fauna (caça ilegal) e do uso de recurso abiótico (extração mineral).

Entre as limitações identificadas no estudo, está a necessidade de maior envolvimento dos gestores com o SAMGe. A reduzida participação dos gestores, pode ser resultado de uma série de fatores: a falta de comunicação entre o ponto focal e gestor da UC, a deficiência no material de orientação, a falta de interesse por parte dos gestores, a falta de crença na continuidade ou efetividade de processos institucionais no geral (WWF, 2017).

De modo geral observa-se que os métodos analisados, são avaliações baseadas principalmente em dados primários de alta subjetividade, coletados por meio de questionários aplicados por meio eletrônico ou por workshops. Estes métodos também apresentam dificuldade em estabelecer correlação entre indicadores mensuráveis e os não mensuráveis. Outra questão, a considerar é a disponibilidade de recursos financeiros que 
influencia diretamente na qualidade dos resultados da avaliação, devido à natureza altamente flexível na implementação das metodologias (STOLL-KLEEMANN, 2010).

Entretanto, a simples avaliação de eficácia da gestão através da aplicação de indicadores e índices, embora necessária, poderá não ser suficiente para atingir os objetivos da área protegida. Para tanto, o envolvimento e as contribuições de vários grupos de partes interessadas podem ser cruciais. Os resultados da avaliação precisam ser considerados e integrados no processo de gestão, para tornar as áreas protegidas mais eficientes (STOLLKLEEMANN, 2010).

Em meio ao desenvolvimento de técnicas e métodos apresentados, verifica-se que os avanços metodológicos observados buscam a integração dos processos de planejamento, gestão e a dinâmica territorial das áreas protegidas, bem como trazem a tona não só resultados práticos, mas também novas abordagens de ação e de efetivação de políticas públicas com resultados reais.

\section{Considerações}

No presente trabalho foi possível observar que mesmo com as mais diversas limitações metodológicas e conceituais, o uso de indicadores e índices para avaliação e planejamento de políticas públicas, vem passando por avanços e podem possibilitar importantes subsídios e efeitos positivos para a otimização do planejamento e gestão de políticas em escalas local, regional e global.

Em linhas gerais a construção das metodologias analisadas, possuem o objetivo de estruturar sistemas que possibilitem a articulação do monitoramento, levando em consideração determinantes e condicionantes ambientais, socioeconômicos e culturais. 0 intuito no fim, é representar a realidade de forma sintética, em diferentes níveis e dimensões. Entretanto, deve-se entender que o efeito das áreas protegidas sobre os valores de conservação só podem ser avaliado ao longo do tempo.

Embora as metodologias analisadas separem a dimensão ambiental da social e econômica, por questão metodológica e conceitual, destaca-se a grande relevância em desenvolver 
analises de forma sistêmica sobre os indicadores, buscando entender as suas inter-relações e limitações estatísticas, que surgem das complexidades e singularidades entre o contexto local e o regional.

Entre os fatores limitantes observados, está a escassez de informações e a descontinuidade na produção de dados estatísticos, o que não permite comparar o desempenho alcançado pelas UCs ao longo do tempo, somado a isso, nota-se grande subjetividade na seleção e ponderação dos indicadores. Outro fator a se destacar é o possível uso restrito dos indicadores em trabalhos acadêmicos e científicos sem o conhecimento dos gestores públicos ou da população, subutilizando as metodologias desenvolvidas, ou mesmo possibilitando seu uso para interesses políticos (MORAES et al., 2016).

Como indicação de pesquisa futura, sugere-se um estudo detalhado, visando à criação de um método com capacidade de mensurar os pontos carentes de ações e medidas necessárias à efetiva governança institucional, social, econômica e ambiental de forma a subsidiar a adoção de políticas públicas efetivas.

Para tanto, é preciso que a metodologia possua variáveis com capacidade de reprodução, com alta confiabilidade e disponibilidade de dados, bem como seja de rápida construção e adaptação com a utilização de fontes primárias e secundárias, o que possibilitaria a redução dos custos e as respostas necessárias para intervenção. Na verdade, o que se propõe é um modo de apresentar simultaneamente o índice sintético e os indicadores por dimensão (ambiental, social, econômica e institucional), de modo que seja possível analisar a interrelação das variáveis de forma agregada e transversal.

\section{Referências bibliográficas}

BANZATO, B. M. Análise da efetividade das unidades de conservação marinhas de proteção integral do estado de São Paulo. Dissertação (Mestrado). Programa de Pós-Graduação em Ciência Ambiental. Universidade de São Paulo. São Paulo. 2014. 161p.

CIFUENTES, M. et al. Medición de la efectividad del manejo de áreas protegidas. WWF: IUCN: GTZ, Turrialba, 2000. 105p.

CLEMENT, F. Analysing decentralised natural resource governance: proposition for a politicised institutional analysis and development framework. Policy Sciences 43. 2010. p. 129- 156. 
ERVIN J. WWF: Rapid Assessment and Prioritization of Protected Area Management (RAPPAM) Methodology. WWF, Gland, Switzerland. 2003. 52 p.

FRANCO NETTO, G.; MIRANDA, A. C. Saúde Ambiental: guia básico para a construção de indicadores. In: BRASIL, Ministério da Saúde. Secretaria de Vigilância em Saúde. Saúde Ambiental: guia básico para a construção de indicadores. Ministério da Saúde. Brasília, 2011. p. 13 - 17.

IZURIETA, A. et al. Medición de la efectividad del manejo de áreas protegidas. WWF - Centroamérica. Turrialba, Costa Rica. 1999. 89p.

ICMBio - Instituto Chico Mendes de Conservação da Biodiversidade. Avaliação comparada das aplicações do método Rappam nas unidades de conservação federais, nos ciclos 2005-06 e 2010. Instituto Chico Mendes de Conservação da Biodiversidade, WWF-Brasil. Brasília: ICMBio. 2011. 134 p.

GETZNER, Michael; JUNGMEIER, Michael; PFLEGER. Bernd. Evaluating Management Effectiveness of National Parks as a Contribution to Good Governance and Social Learning. InTech/Open Science. Protected Area Management. 2012. p. $129-146$.

GELDMANN, J. et al. Mapping change in human pressure globally on land and within protected areas. Conservation Biology, 28. 2014. p. 1604-1616.

GELDMANN, J. et al. A global analysis of management capacity and ecological outcomes in terrestrial protected areas. Conservation Letters. 2017. p. $1-10$.

HOCKINGS, M.; STOLTON, S.; LEVERINGTON, F.; DUDLEY, N.; COURRAU, J. Evaluating Effectiveness: A framework for assessing management effectiveness of protected areas. IUCN, Gland, Suíça, Cambridge, Reino Unido. 2006.

HOCKINGS, M. et al. Protected area management effectiveness. In: Worboys, Lockwood, Kothari, Feary, Pulsford (eds). Protected Area Governance and Management. ANU Press, Canberra. 2015. p. 889 - 928.

LEVERINGTON, F. et al. A Global Analysis of Protected Area Management Effectiveness. Environmental Management. 2010.

NELSON, A. K.; CHOMITZ. M. Effectiveness of Strict vs. Multiple Use Protected Areas in Reducing Tropical Forest Fires: A Global Analysis Using Matching Methods. PLOS ONE. Volume 6. Issue 8. 2011. p. 01-14

JANNUZZI P.M. Indicadores sociais no Brasil. Campinas: Alínea. (2006). 141p.

JUFFE-BIGNOLI D, et al. Protected Planet Report 2014: Tracking progress towards global targets for protected areas. UNEP-WCMC, Cambridge, UK. 2014.

JÚNIOR, L. C. de A.; AGRA FILHO, S. S. Estudo comparativo entre três diferentes métodos de avaliação da efetividade de gestão de áreas protegidas. Revista GESTA. v. 3, n. 1. 2015. p. 232-241.

LEMOS DE SÁ, R. Unidades de conservação: espaços ameaçados ou áreas protegidas. (Relatório Técnico). Brasília. 2000. 32 p.

MARINELLI, C. E. De olho nas unidades de conservação: Sistema de Indicadores Socioambientais para Unidades de Conservação da Amazônia Brasileira. Instituto Socioambiental. São Paulo. 2011. 12. p.

MORI, K.; CHRISTODOULOU. A. Review of sustainability indices and indicators: Towards a new City Sustainability Index (CSI). Environmental Impact Assessment Review 32. 2012. p. 94 -106.

MORAES, D. E.; CARVALHO, M. A.; CANÔAS, S. S. Indicadores sintéticos de qualidade de vida e o conceito de natureza/ambiente. Caminhos de Geografia Uberlândia v. 17, n. 58. 2016. p. 123-135.

PADOVAN, M. P. Formulacion de um estandar y um procedimento para la certificacion del manejo de áreas protegidas. Centro Agronomico Tropical de Investigacion y Ensenanza. Turrialba, Costa Rica. 2001.

PADOVAN, M. P. Certificação de Unidades de conservação. São Paulo: conselho nacional da reserva da Biosfera da Mata Atlântica. Ministério do Meio Ambiente, Unesco- MaB, secretaria de estado do Meio Ambiente de são Paulo. 2003. 55p. 
PADUA, C. V.; CHIARAVALLOT, R. M. Pesquisa e conhecimento na gestão de unidades de conservação. Org: CASES. M. O. Gestão de Unidades de Conservação: compartilhando uma experiência de capacitação. WWFBrasil/IPÊ-Instituto de Pesquisas Ecológicas. WWF-Brasil, Brasília, 2012. p. 139 - 153.

SILVA, C. M. da. Estratégias para implementação de governança ambiental no refúgio de vida silvestre Mata do Junco, uma unidade de conservação estadual de Sergipe. Dissertação (Mestrado em Desenvolvimento e Meio Ambiente). Universidade Federal de Sergipe. São Cristóvão. 2016. 194p.

SOBRAL, A. et. al. Definições básicas: dado, indicador e índice. In: BRASIL, Ministério da Saúde. Secretaria de Vigilância em Saúde. Saúde Ambiental: guia básico para a construção de indicadores. Ministério da Saúde. Brasília. 2011p. 25 - 52.

STOLTON S.; HOCKINGS, M.; DUDLEY, N.; MACKINNON, K.; WHITTEN, T.; LEVERINGTON; F. Reporting Progress i $\mathrm{n}$ Protected Areas a Site-Level Management Effectiveness Tracking Tool: second edition. World Bank/WWF Fo rest Alliance published by WWF, Gland, Switzerland. (2007).

STOLL-KLEEMANN, S. Evaluation of management effectiveness in protected areas: Methodologies and results. Basic and Applied Ecology 11. 2010. p. 377-382.

WILKIE, D. S. et al. Parks and people: assessing the human welfare effects of establishing protected areas for biodiversity conservation. Conservation Biology: the journal of the Society for Conservation Biology, v. 20, n. 1, 2006. p. 247-249.

WWF - World Wide Found for Nature Brasil. Áreas Protegidas ou Espaços Ameaçados: O Grau de Implementação e a vulnerabilidade das Unidades de Conservação federais Brasileiras de Uso Indireto. Org. DE SÁ, Rosa M. Lemos; FERREIRA, Leandro. Brasília. 1999. p. 33.

WWF-BRASIL; SEMARH-GO. Implementação da avaliação rápida e priorização da gestão de unidades de conservação (Rappam) em unidades de conservação estaduais em Goiás. Brasília, DF. 2014. 106p.

WWF Brasil - World Wide Found for Nature. Avaliação da gestão das unidades de conservação: métodos RAPPAM (2015) e SAMGE (2016). WWF Brasil. 1a ED. Brasília. 2017. 127p. 